\title{
El empleo de de repente como introductor de la complicación en narraciones de experiencia personal de hablantes de Santiago de Chile
}

\author{
Silvana Guerrero \\ Universidad de Chile \\ Javier González \\ Pontificia Universidad Católica de Chile
}

\section{RESUMEN}

En el marco del variacionismo, en este trabajo se realiza una aproximación sociolingüística al empleo de de repente como introductor de la complicación de la narración en una muestra de 300 narraciones de experiencia personal (Labov y Waletzky 1967, y Labov 1972) de hablantes santiaguinos. Primero, se establece la frecuencia de empleo de dicha partícula. Luego, se correlacionan los factores sociodemográficos de los hablantes con el empleo de de repente y, finalmente, se describe el funcionamiento de esta partícula en las narraciones que conforman la muestra. La hipótesis de trabajo fue que las características sociodemográficas de los hablantes de español en Santiago de Chile están correlacionadas con el empleo de de repente como introductor de complicación de la narración. El análisis de los datos dio cuenta de que de repente tiene una frecuencia de 100 casos, de los cuales 95 (95\%) cumplen la función de introducir la acción complicada de los relatos. Asimismo, demostramos que se trata de una partícula

https://doi.org/10.18800/lexis.201701.002 
que suele emplearse más por informantes mujeres de edad joven (20-34 años). El análisis, a su vez, permitió dar cuenta de que una colocación preponderante es la que se genera entre la forma de repente + presente histórico.

Palabras clave: sociolingüística variacionista, variación discursiva, narraciones de experiencia personal, de repente

\section{AbSTRACT}

Situated within the variationist sociolinguistic framework, in this paper we analyze the use of de repente as a marker that introduces complication or climax in a sample of 300 personal experience narratives (Labov y Waletzky 1967 y Labov 1972) of Santiago speakers. First, we establish the frequency of use of this particle. Then, we correlate the speakers' sociodemographic factors with the use of this marker, and finally, we describe the functionality of this particle within the narratives that make up the sample. Our hypothesis was that the sociodemographic characteristics of Santiago Spanish speakers would be correlated with the use of de repente as a phrase that introduces complication or climax within the narrative. The data analysis shows that de repente was used a total of 100 times, of which $95(95 \%)$ function in this manner, or as introducing the complicated action of the story. Additionally, we demonstrate that this particle is used most frequently by female informants from the younger age group (20-34 years old). The analysis, in turn, enabled us to determine that a main collocation is that of de repente + historical present.

Keywords: variationist sociolinguistics, discourse variation, personal experience narratives, de repente 


\section{Introducción}

\subsection{Naturaleza, alcance y objetivos del estudio}

No cabe duda de que la sociolingüística ha tenido la oportunidad de conocer muy de cerca los procesos y mecanismos de la interacción comunicativa. Dichos procesos de interacción proporcionan un resultado tangible, esto es, un producto de lengua hablada del que la sociolingüística ha reunido muestras muy bien nutridas y que suele tener la forma de conversaciones o diálogos, mantenidas entre los investigadores y sus informantes (Moreno Fernández 2009). En este contexto, Johnstone (2006) destaca que la narrativa oral ha sido objeto de múltiples investigaciones, la mayoría de ellas centradas en su estructura más que en la manera en que la estructura y las funciones propias de la narrativa podrían influir en la variación y el cambio lingüístico. Lo que esta autora propone es que la estructura y la función de la narrativa, en tanto proceso de construcción y narración resultante, pueden arrojar luces sobre un tema de interés central para los variacionistas, a saber, la formación del vernáculo, en el sentido de que podrían existir conjuntos particulares de rasgos lingüísticos propios de las comunidades de habla.

Tomando en consideración que el análisis sobre las narrativas orales en el español hablado en Chile es todavía escaso, en particular, desde el punto de vista sociolingüístico, en esta investigación queremos poner el énfasis en analizar de manera exclusiva los condicionamientos lingüísticos en la construcción de narraciones de experiencia personal. Entre ellos, es de especial interés el estudio de de repente como introductor de complicación de la narración. La partícula de repente ha sido definida por el Diccionario de la Lengua Española (2001) como una locución adverbial equivalente a "súbitamente, sin preparación, sin discurrir o pensar" o como una locución adverbial coloquial que alude a "posiblemente" en países como Uruguay y Venezuela (s.v. repente).

De manera concreta, en este trabajo se realiza una aproximación sociolingüística, en el marco del variacionismo, al empleo del de repente como introductor de la complicación de la narración en 
una muestra de 300 narraciones de experiencia personal (Labov y Waletzky 1967, y Labov 1972). 192 de dichas narraciones fueron extraídas del corpus del grupo de Estudio Sociolingüístico del Español de Chile (ESECH) y 108 del Corpus del Proyecto para el Estudio Sociolingüístico del Español de España y América (PRESEEA) correspondiente al habla de Santiago de Chile. El objetivo general de este estudio es analizar el comportamiento de de repente a nivel discursivo y su estratificación sociolingüística en el corpus descrito. Nuestros objetivos específicos son, primero, establecer la frecuencia de empleo de dicha partícula. Luego, comprobar si existe variación entre los factores sociodemográficos de los hablantes y el empleo de de repente como partícula introductoria de la complicación de la narración y, finalmente, describir el funcionamiento de esta partícula en las narraciones de experiencia personal de los hablantes santiaguinos.

La hipótesis que guía nuestro trabajo es que las características sociodemográficas de los hablantes de español en Santiago de Chile están correlacionadas con el empleo de de repente como introductor de complicación de la narración. Para proponer esta hipótesis, nos basamos en el trabajo doctoral de Guerrero (2014a), donde se estudió la variación discursiva en narraciones de experiencia personal en el español hablado en Santiago de Chile. En dicho trabajo, los condicionamientos lingüísticos en la construcción de los relatos tienen menos protagonismo que los condicionamientos sociales, por lo que, para el desarrollo de esta investigación, hemos optado por centrarnos en un elemento particular que fue descrito someramente en el trabajo mencionado, la partícula de repente.

En este contexto, destacamos que los estudios que han abordado el funcionamiento de de repente, lo han hecho más bien desde el punto de vista descriptivo, en tanto marcador discursivo. Asimismo, hay aportaciones desde la semántica y desde el análisis del discurso, pero ninguno de estos estudios ha analizado su comportamiento en secuencias discursivas mayores y menos desde el punto de vista sociolingüístico. Recientemente, González (2013) analizó el empleo de esta partícula, junto con otras, en el español caraqueño, 
en tanto operador de concreción. ${ }^{1}$ Por lo anterior, este trabajo no solo contribuye a la descripción teórica, sino que se perfila como una contribución a la descripción sociolingüística del español hablado en Santiago de Chile.

\section{Marco conceptual}

2.1. De repente y su aplicación en estudios de variación sociolingüística

Como ya mencionáramos, en el marco del variacionismo laboviano (Labov 1983 [1972]), en esta investigación nos proponemos analizar el empleo de de repente en una muestra de discurso natural de hablantes santiaguinos, con lo que, además, esperamos contribuir al estudio del registro coloquial de la lengua española. Como sabemos, la variación puede manifestarse en los distintos niveles de la lengua, esto es, fonético-fonológico, morfosintáctico, léxico y pragmático-discursivo. Moreno Fernández (2012) sostiene que el uso lingüístico se convierte en un concepto clave para explicar la variación, ya que, por una parte, determina y refleja la variabilidad interna y, por otra, es sensible a la diversidad de condicionamientos externos, adecuándose a ella. Sin embargo, la variación pragmático-discursiva ha sido una de las menos tratadas en los estudios sociolingüísticos, por lo que es necesario comenzar a incorporar varios factores a la descripción lingüística, en lo fundamental, lo pragmático, lo social, lo cognitivo, lo comunicativo y lo contextual (Serrano 2011). En este estudio, el eje fundamental es el vínculo

1 González (2013: 63) propone el siguiente ejemplo: “[El campo] Es más tranquilo porque no tienen ninguna clase de problema. No tienen problemas de... de... de repente (/ por ejemplo, \# repentinamente, \# quizás, \# a veces) de llegar a un horario de un trabajo, o que el jefe te esté presionando. (CB3MB. 87, 165)”. A nuestro juicio, si bien la forma de repente podría alternar con por ejemplo, también podría alternar con quizás, que la autora considera como inapropiada en este contexto. En el ejemplo, quizás podría intercambiarse con de repente, pues la repetición de "de" por parte del hablante indica una posible duda respecto de lo que va a señalar. Consideramos, en este sentido, que el ejemplo que proporciona la autora es discutible. 
entre lo discursivo-pragmático y lo social, pues el fenómeno que nos atañe ejerce una función trascendental en el discurso.

Junto con lo anterior, en esta investigación partimos de la base de que gran parte de los hechos de variación lingüística varían en forma sistemática, si se los correlaciona con variables sociodemográficas como el grupo socioeconómico, ${ }^{2}$ la edad y el sexo de los informantes. Esto implica que el hecho de que las variables lingüísticas estén en covariación sistemática con las variables externas permite a los individuos instalarse en un determinado espacio del espectro social. En relación con esto, Coates (2009) pone de relieve que "aunque desde el punto de vista lingüístico las variables lingüísticas sean formas equivalentes para referirse a algo en particular, son diferentes desde una perspectiva social” (89). Por su parte, Moreno Fernández (1998) y Blas Arroyo (2005) también señalan que no es posible reconocer de antemano qué tipo de variables sociales van a actuar sobre la variación lingüística, primero, porque la variabilidad no tiene por qué manifestarse de la misma forma en comunidades de habla diferentes y, segundo, porque estos mismos factores no se configuran de la misma forma en sociedades distintas. En nuestro caso, los factores sexo, edad, grupo socioeconómico y nivel educacional serán las variables independientes empleadas en esta investigación sobre el carácter que asume la variación lingüística correspondiente al empleo del de repente en una muestra del español hablado en Santiago de Chile.

\subsection{Caracterización de de repente}

Las caracterizaciones que se han realizado sobre la partícula de repente han sido, en su mayoría, con base en una perspectiva descriptiva. Al respecto, la Real Academia Española (2010) la considera como una locución adverbial, debido a que cumple una función de modificación de sustantivos, adjetivos u otros adverbios. Destaca, además, que, dado su grado de fijación, no se trata de un grupo

\footnotetext{
2 En esta investigación trabajaremos tanto con la variable grupo socioeconómico como con la variable nivel educacional (cf. 3.1.1. y 3.1.2.)
} 
preposicional y, asimismo, señala que su significado de "repentinamente" es compartido en todas las variedades del español, aunque en algunos países de América y en las Islas Canarias también es conmutable por quizá, a lo mejor, posiblemente. En tanto locución adverbial, dicha caracterización es compartida por Alarcos Llorach (1999), quien enfatiza, a su vez, la función de este tipo de locuciones como adyacentes circunstanciales. Para el caso de Chile, el Diccionario de Uso del Español de Chile (DUECh 2010) señala que de repente puede ser considerado como una locución adverbial "de frecuencia esporádica”, o bien como un marcador discursivo que indica que lo que se dirá debe ser interpretado como probable, pero incierto para el hablante (s.v. repente/ de repente).

Desde la gramática del texto, de repente es descrito como un marcador discursivo, debido, en lo fundamental, a que cumple atributos destacados por autores como Gili-Gaya (1961) y Portolés (2001), a saber, que como unidad lingüística permita continuidad y transición en el discurso, que sea relativamente invariable desde el punto de vista sintagmático y que permita guiar inferencias en el proceso de comunicación. Se trata, en concreto, de un modalizador epistémico que cumple la función de atenuar la fuerza argumentativa de lo enunciado.

Sobre esta última aproximación, en el habla chilena se ha caracterizado el rol de los marcadores discursivos desde la funcionalidad pragmática que cumplen en el discurso, reconociéndose que por medio de su uso los hablantes manifiestan su subjetividad en sus enunciados. En esta línea, Becerra et al. (2005) analizan el empleo de los marcadores discursivos utilizados por hablantes de Santiago de Chile y caracterizan a la partícula de repente como un modalizador - en tanto implica una marcación de subjetividad por parte del hablante- de atenuación, ya que indica inseguridad epistémica. Esta observación de atenuador es compartida por Briz et al. (2008), quienes señalan que en Argentina, Chile y México la referida partícula discursiva mitiga la fuerza de aquello que se expresa, de manera que atenúa su fuerza argumentativa. 
Junto con lo anterior, uno de los trabajos más interesantes para el español actual de Santiago de Chile es el de Rojas (2008), quien señala que existen tres funciones semánticas para la partícula de repente. La primera función (DR1) corresponde a un evento concebido como súbito e inesperado. La segunda (DR2) alude a un evento concebido como poco frecuente, y la tercera (DR3) es la emisión del hablante concebida como atenuada en su fuerza argumentativa. En todos estos casos, el hablante, por medio del uso de esta partícula, aporta información con respecto a su subjetividad, es decir, implica sus creencias acerca de la probabilidad de que algo pueda ocurrir o que aquello que enuncia sea cierto. En esta misma pesquisa se hace alusión a un cambio semántico si se analiza el uso de la partícula en cuestión desde una perspectiva diacrónica. En esta evolución han sido procesos metafóricos y metonímicos los que han configurado su gramaticalización, la que propende a un lineamiento DR1 $>$ DR2 $>$ DR3, lo que implicaría una tendencia al uso de de repente como atenuador de fuerza argumentativa (DR3).

\subsection{De repente en el marco de la narración de experiencia personal}

Como ya se señaló, situamos nuestro estudio de de repente en la narración de experiencia personal. La bibliografía sobre narraciones es extensa; no obstante, las investigaciones pioneras de Labov y Waletzky (1967) y de Labov (1972) han demostrado ser el modelo de más alto rendimiento empírico en la investigación sobre el problema, como ha quedado de manifiesto en varios estudios sobre narrativas (Reyes 1996, 2002, 2003; Soler 2004; Jiménez 2006; y Shiro 2003, por solo citar algunos ejemplos). Labov (1972: 360) define la narración como "one method of recapitulating past experience by matching a verbal sequence of clauses to the sequence of events which (it is inferred) actually occurred [un método de recapitulación de la experiencia pasada adecuando una secuencia verbal de proposiciones a la secuencia de sucesos que (se supone) ocurrieron realmente (la traducción es nuestra)]”. La narración es considerada como un texto que expresa una serie de eventos que ocurren en el tiempo, pero donde, a su vez, se percibe de manera subyacente 
una relación causal o temática. Labov y Waletzky (1967) y Labov (1972) distinguen dos tipos de relatos: los relatos mínimos, compuestos de - al menos- dos cláusulas narrativas en pasado y los relatos completos. Dichos autores plantean que una narración de experiencia personal plenamente formada exhibe seis rasgos estructurales bien definidos, a saber: 1) resumen, 2) orientación, 3) acción de complicación de la narración, 4) evaluación, 5) resultado o resolución y 6) coda o moraleja. Para los fines de esta investigación, la importancia de los aportes del trabajo de Labov y Waletzky (1967) y del de Labov (1972) radica en que la partícula de repente ha sido descrita como aquella que introduce un hecho inesperado o dramático (Silva-Corvalán 2001).

El hecho inesperado corresponde precisamente a la complicación de la narración, definida por Labov y Waletzky (1967: 354-396) y Labov (1972: 360) como el núcleo o esqueleto de la narración en el que aparecen los distintos eventos narrados, constituyendo, de esta forma, el clímax del relato. Dicha parte de la narración se caracteriza por incorporar cláusulas narrativas. Al respecto, Bassols y Torrent (2003: 169) señalan que en todo relato debe existir una complicación o problematización; no es un proceso rutinario el que da lugar a una narración, aunque incluya un conjunto de acciones vinculadas por la lógica de causa-efecto, sino que la sucesión de acontecimientos debe ser alterada por algún hecho inesperado que provoque una desviación en el curso normal de las cosas. Así, en este trabajo sugerimos que la narración de los acontecimientos inesperados es frecuentemente introducida por la expresión de repente.

\section{Metodología}

\subsection{Corpus}

El corpus que sirvió de base para el análisis del presente estudio consistió en 300 narraciones de experiencia personal extraídas de dos corpus, a saber, el corpus del grupo de Estudio Sociolingüístico del Español de Chile (ESECH) y el corpus del Proyecto para el Estudio Sociolingüístico del Español de España y América (PRESEEA) de 
Santiago de Chile. ${ }^{3}$ Para la recopilación de ambos corpus, se aprovechó la instancia de aplicación de un instrumento que se realiza en el marco de la asignatura de Sociolingüística que se imparte en las Licenciaturas en Lengua y Literatura Hispánica, y Lengua y Literatura Inglesas de la Universidad de Chile. Las entrevistas fueron hechas a hombres y mujeres con características sociodemográficas congruentes, aplicando los supuestos metodológicos sugeridos por Labov (1983 [1972]). Consecuentemente, en dichas situaciones los entrevistadores debían tratar de superar la "paradoja del observador"4 consiguiendo, de esta forma, una muestra significativa de discurso natural grabado (vernáculo) ${ }^{5}$ de hablantes de la comunidad de habla en estudio.

3 El ESECH es un grupo de estudio integrado por académicos y estudiantes del Departamento de Lingüística de la Facultad de Filosofía y Humanidades de la Universidad de Chile. El objetivo general del proyecto es estudiar la influencia de factores sociales y contextuales en el uso lingüístico de la lengua española en Chile. El equipo cuenta con un corpus de 192 entrevistas sociolingüísticas estratificadas sociodemográficamente, recopiladas entre 2008 y 2012. Por su parte, el PRESEEA es un proyecto para la creación de un corpus de lengua española hablada representativo del mundo hispánico en su variedad geográfica y social. Esos materiales se reúnen atendiendo a la diversidad sociolingüística de las comunidades de habla hispanohablantes. En la actualidad, el proyecto agrupa a cerca de 40 equipos de investigación sociolingüística. El equipo de PRESEEA en Santiago de Chile está compuesto por los profesores de la Universidad de Chile, Alfredo Matus Olivier, Abelardo San Martín Núñez y Silvana Guerrero González, y está conformado por 108 entrevistas sociolingüísticas, recopiladas entre 2007 y 2012.

4 Según Labov (1983 [1972]), el objetivo de la investigación lingüística de una comunidad de habla consiste en estudiar la forma en la que la gente habla cuando no está siendo sistemáticamente observada; sin embargo, solo podemos obtener tales datos mediante la observación sistemática. A esto es a lo que se le ha denominado paradoja del observador. Este dilema se soluciona encontrando la manera de completar la entrevista formal con otros datos, o cambiar la estructura de la situación de la entrevista de una u otra manera. En este sentido, una forma de superar esta paradoja consiste en romper las constricciones de la situación de entrevista mediante diversos procedimientos que distraen la atención del sujeto en su propio discurso y pueden hacer brotar su habla más vernácula.

5 Citando a Labov, Moreno Fernández (2012: 181-182) señala que: “El vernáculo, en el que se presta al discurso propio una atención mínima, aporta los datos más sistemáticos por el análisis lingüístico. El vernáculo se define como la forma de hablar adquirida durante la preadolescencia”. 
El cuestionario utilizado para la realización de las mencionadas entrevistas consta de varias secciones, entre ellas, la que permite obtener las secuencias discursivas que conforman el corpus de nuestra investigación. En relación con esto, es necesario precisar que se seleccionó la narración mejor desarrollada por cada informante. De esta forma, se pudo medir el mejor desempeño de cada uno de ellos y, posteriormente, compararlos. Este criterio de selección de las secuencias mejor desarrolladas nos permitió controlar algunas variables extrañas, como el cansancio y el desinterés de los sujetos considerando la duración de la entrevista, entre otras que pudieran surgir en el marco de la misma.

\subsubsection{Caracterización de la muestra}

La muestra analizada consta de 300 narraciones de experiencia personal generadas por hombres y mujeres de Santiago de Chile. En lo referido a los tres grupos etarios que hemos considerado apelamos a tres momentos vitales (Blas Arroyo 2005), es decir, en el primer grupo (20 a 34 años) se encuentran los sujetos que ingresan al mundo laboral o que llevan poco tiempo desempeñando una profesión o actividad específicas, pues a partir de ese momento hay un desarrollo de los patrones "adultos" de variación. Por su parte, en el segundo grupo (35 a 54 años) están incluidos los sujetos que tienen un desarrollo laboral pleno y, finalmente, en el tercer grupo etario (55 años y más) se hallan los sujetos que están prontos a dejar el mundo laboral o que ya lo han dejado. En este sentido, se trabaja con etapas por las que pasa el hablante, que suelen estar vinculadas con la cultura de cada comunidad lingüística (Serrano 2011).

Para la estratificación de los sujetos que conforman la muestra del ESECH, se empleó el sistema de adscripción de estatus social elaborada por el equipo, que parte de una asignación de puntaje según las siguientes tres variables: 1) nivel educacional, 2) profesión u ocupación y 3) comuna de residencia. A cada una de estas variables se le otorgó una ponderación distinta, a saber: 3 puntos para el nivel educacional, 2 para la variable profesión u ocupación y 1 punto para la variable comuna de residencia. La escala incluye cuatro estratos 
socioeconómicos, que son los siguientes: bajo, medio bajo, medio y medio alto, para cuya diferencia se cauteló la consistencia entre las variables antes mencionadas de manera que respondieran a lo que Lenski (1954) denomina congruencia de estatus ${ }^{6}(c f$. San Martín y Guerrero 2015).

Por su parte, siguiendo el sistema de estratificación del Proyecto PRESEEA, se estratificó la segunda parte de la muestra de sujetos de acuerdo con sus respectivos niveles de instrucción, es decir, según la equivalencia entre los niveles de estudios básico, secundario y superior con los niveles bajo, medio y alto, respectivamente. Sin embargo, con el propósito de garantizar la homogeneidad en la composición de los distintos estratos de la muestra y, por lo tanto, la representatividad de los mismos en relación con la población analizada, se complementó dicho sistema de estratificación con el procedimiento de estratificación empleado por el proyecto de Estudio Sociolingüístico del Español de Chile (ESECH).

\subsubsection{Población y muestra}

Para la conformación del corpus se consideró la población santiaguina. ${ }^{7}$ El cuestionario de la entrevista se aplicó a una muestra de sujetos del tipo denominado "muestra por cuotas con afijación uniforme” (López Morales 1994). La muestra así conformada comprende un total de 300 individuos, distribuidos proporcionalmente como se indican las tablas 1 y 2, que se incluyen a continuación.

\footnotetext{
6 Según Lenski (1954), se considera que un individuo es congruente con su estatus cuando las puntuaciones obtenidas en las diferentes dimensiones usadas para medir el estatus, son más o menos iguales, independiente de que sus rangos sean altos, bajos o estén en una extensión media. Cuando las puntuaciones del individuo son muy diferentes, se habla de incongruencia de estatus.

7 Por lo que refiere a los criterios de asignación de hablante nativo de Santiago de Chile, de acuerdo con Prieto (1995-1996: 399), se aplicaron las siguientes restricciones en la selección de los sujetos: 1) haber nacido y residido en forma ininterrumpida en Santiago, 2) haber residido en forma ininterrumpida en Santiago desde los cinco años de edad y 3) haber nacido en Santiago y haber residido en Santiago la mayor parte de sus vidas, salvo por periodos que sumados no superen los cuatro años en el tramo de 55 años y más, y los tres años en el tramo de 35 a 54 años.
} 
Tabla 1. Distribución de sujetos según características sociodemográficas de sexo, edad y grupo socioeconómico, según ESECH

\begin{tabular}{lccccccc}
\hline \multicolumn{1}{c}{ Grupo de edad } & \multicolumn{2}{c}{$20-34$} & \multicolumn{2}{c}{$35-54$} & \multicolumn{2}{c}{55 y más } & \multirow{2}{*}{ Total } \\
\cline { 1 - 7 } Grupo socioeconómico & $\mathrm{H}$ & $\mathrm{M}$ & $\mathrm{H}$ & $\mathrm{M}$ & $\mathrm{H}$ & $\mathrm{M}$ & \\
\hline Medio alto & 8 & 8 & 8 & 8 & 8 & 8 & $=48$ \\
Medio & 8 & 8 & 8 & 8 & 8 & 8 & $=48$ \\
Medio bajo & 8 & 8 & 8 & 8 & 8 & 8 & $=48$ \\
Bajo & 8 & 8 & 8 & 8 & 8 & 8 & $=48$ \\
\hline & 32 & 32 & 32 & 32 & 32 & 32 & $=192$ \\
\hline
\end{tabular}

Tabla 2. Distribución de sujetos de la muestra por nivel educacional, sexo y edad, según PRESEEA

\begin{tabular}{|c|c|c|c|c|c|c|c|}
\hline Grupo de edad & \multicolumn{2}{|c|}{$20-34$} & \multicolumn{2}{|c|}{$35-54$} & \multicolumn{2}{|c|}{55 y más } & \multirow{2}{*}{ Totales } \\
\hline Nivel educacional & $\mathrm{H}$ & M & $\mathrm{H}$ & M & $\mathrm{H}$ & M & \\
\hline Alto & 6 & 6 & 6 & 6 & 6 & 6 & 36 \\
\hline Medio & 6 & 6 & 6 & 6 & 6 & 6 & 36 \\
\hline Bajo & 6 & 6 & 6 & 6 & 6 & 6 & 36 \\
\hline Totales & 18 & 18 & 18 & 18 & 18 & 18 & 108 \\
\hline
\end{tabular}

\subsection{Procedimiento analítico}

De acuerdo con los objetivos trazados en esta investigación, el análisis tuvo, en lo primordial, cuatro etapas: 1) selección de las 300 narraciones de experiencia personal que conforman la muestra, 2) determinación de la frecuencia de empleo de de repente en cada caso, 3) correlación entre la partícula en estudio con los factores sociodemográficos de los hablantes en cada corpus y 4) descripción del funcionamiento de de repente en narraciones de experiencia personal en hablantes de Santiago de Chile. De este modo, pudimos cumplir los objetivos propuestos para este estudio. 
En lo que respecta a la descripción de los procedimientos estadísticos aplicados, en esta investigación incluiremos tanto el análisis descriptivo (frecuencias absolutas y los porcentajes respectivos) como el inferencial. En este último caso, el paquete estadístico empleado es el SPSS (Statistical Package for the Social Sciences), versión 15.0. El grado de significación se definirá en el 5\%, según el cual $\mathrm{p}<0,05$ será estadísticamente significativo. Dichos procedimientos siguen las sugerencias de Moreno Fernández (1990), López Morales (1994) y Hernández Campoy y Almeida (2005).

\section{Presentación y análisis de los resultados}

A continuación presentamos los hallazgos relevados en este estudio. Para mayor claridad en esta presentación, distinguiremos, en los ejemplos extensos, cada parte de la estructura narrativa con un sistema de etiquetas de inicio $<>$ y de cierre $</>$ y subrayaremos los elementos evaluativos, transversales en la narración, que es transliterada en ortografía convencional. La partícula en estudio aparece ensombrecida. Al final de cada ejemplo aparece el corpus específico del cual se extrajo y el código del hablante en dicho corpus. ${ }^{8}$

\subsection{Análisis general del empleo de de repente en narraciones de experiencia personal de hablantes santiaguinos}

En las 300 narraciones analizadas, la partícula de repente aparece en 100 ocasiones. De este total, solo en 5 (5\%) casos la partícula no

8 En el corpus PRESEEA de Santiago de Chile (SCHI), el código es el siguiente: $\mathrm{H}=$ Hombre y $\mathrm{M}=$ Mujer. El primer número, luego del sexo del informante, corresponde al grupo etario: $1=20-34$ años; $2=35-54$ años y $3=55$ años y más. El segundo número corresponde al nivel educacional del informante: $1=$ estudios básicos completos o incompletos; $2=$ estudios medios completos o incompletos y $3=$ estudios universitarios completos o incompletos. El número que sigue al guion corresponde al número correlativo que reciben los sujetos que conforman la muestra. En el corpus ESECH, la primera letra corresponde al grupo socioeconómico: $\mathrm{B}=\mathrm{B}$ ajo, $\mathrm{MB}=$ Medio bajo, $\mathrm{M}=$ Medio y $\mathrm{A}=$ Alto; el número romano que sigue corresponde al grupo etario del hablante, esto es, I= 20-34 años; II= 35-54 años y III= 55 años y más; $\mathrm{H}=$ Hombre y $\mathrm{M}=$ Mujer. El número que sigue al guion corresponde al número correlativo que reciben los sujetos que conforman la muestra. 
figura como introductora de cláusulas correspondientes a la complicación de la narración. En las 5 ocasiones de repente adquiere el valor que Rojas (2008) denomina DR2, esto es, una locución adverbial de frecuencia, equivalente a "a veces", "de vez en cuando", "en ciertas ocasiones", como vemos en los ejemplos correspondientes.

(1) yo siempre viví pensando en mi madre / sabía que tenía a mi madre / sabía que tenía dos hermanos una mayor y uno menor que yo / y pascuas tristes porque veía yo otras familias que estaban con sus hijos / que regalos / que pequeños regalos de repente / qué sé yo / y yo sin / sin mi madre que era lo que más deseaba / siempre deseé conocerla / tenerla (...) (PRESEEA: H21_037).

(2) (...) y dice que habían dos señoras / dos viejitas // vivían solitas / y siempre le rezaban a las ánimas del purgatorio las señoras / entonces / de repente bajaban cuatreros (...) (PRESEEA: M31_032).

(3) (...) porque ya no es el que yo quiera tomar la micro / vamos a ver si la micro pasa// yo de repente de mi trabajo de la casa al trabajo / me demoro treinta minutos si es que no hay taco // E.: ya//I.: pero en este minuto me demoro lo mismo / siempre y cuando pueda tomar la micro / porque a veces tengo que esperar hasta treinta minutos / cuarenta y hasta una hora he esperado que una micro pase o que se digne a parar / porque vienen llenas y obviamente que no te paran de repente los choferes hay paraderos establecidos y los choferes tampoco lo respetan porque a veces van apurados o se han demorado mucho y no tienen ningún tipo de respeto con la frecuencia (...) (PRESEEA: M22_058).

(4) (...) éramos más/ éramos más pavos/ más más sanos que ahora/ o sea/ de repente nos podíamos tomar un trago de cerveza (...) (ESECH: BII_H017).

Como puede apreciarse en los ejemplos precedentes, de repente se ubica en cláusulas que corresponden a la orientación de la historia. 
Este hallazgo comprueba lo que señala el DUECh (2010), pero, como vemos, se trata de un uso muy poco recurrente en este tipo de tipología discursiva. En consecuencia, su empleo parece estar determinado, en parte, por el tipo de discurso en el que se inserta.

Los 95 casos restantes (95\%) corresponden a lo que Rojas (2008) denomina DR1, es decir, una locución adverbial de modo, equivalente a "súbitamente", “de manera repentina”. En todos los casos, la partícula en estudio cumple la función de introducir cláusulas narrativas correspondientes a la complicación del relato. Del total de casos, 44 (46,3\%) corresponden al corpus del PRESEEA de Santiago de Chile y $51(53,7 \%)$ al corpus del proyecto ESECH. Este último dato contribuye parcialmente a nuestro estudio, porque el corpus del ESECH es notoriamente más grande que el del PRESEEA de Santiago de Chile, por lo tanto, los resultados eran esperados. No obstante, explicitamos dicha información dado que será útil en la segunda parte de este análisis.

A continuación, presentaremos ejemplos que dan cuenta de cómo funciona la partícula de repente en la acción de complicación de los relatos que conforman nuestra muestra de estudio.

(5) 9

a. I: (...) <acción de complicación> y de repente un huevón de dieciocho años y yo teniendo ocho años el huevón muy maricón / me dijo eeh ¡ya huevón eeh hay que entrar hay que entrar! / para hacer/ y se estaba haciendo el lindo con una mina el huevón ¿cachái? así como para hacerse el bacán

b. y voy corriendo

c. y hay un ventanal / de vidrio

d. y yo era el único que no había entrado po

e. y cuando voy a entrar

f. el huevón antes de entrar me cierra el ventanal

9 Dada la extensión de este relato, y del que expondremos posteriormente, hemos seleccionado solo aquellos segmentos discursivos que nos permiten ejemplificar el comportamiento de la partícula discursiva en estudio. 
g. pero yo soy tan terco y de chico lo he sido /

h. soy orgulloso

i. seguí corriendo

j. y traspasé el vidrio así jtaa! así / onda lo traspasé

k. salté y lo traspasé

1. y no me pasó nada

m. pero cuando estaba cayendo / el vidrio que estaba en el suelo me resbalé

n. y me enterré un vidrio atrás de de la rodilla así (...)

o. y se me cayó la pantorrilla para atrás / o sea como la parte de atrás de esto

p. y se me fue para atrás ipf!

q. y se me veía el tendón

r. y yo pesqué un cuchillo o sea no un cuchillo un vidrio

s. y lo salí persiguiendo así ¡guaah! llorando

t. y el huevón / eeh corrió así desesperado

u. y subió a un segundo piso / había una escalera por ahí

v. y la cosa eh que cuando va a subir la escalera

w. yo empiezo a subir la escalera y no podía

x. empecé a cojear ¿̨cachái? no podía

y. y una señora / como que me vio

z. y cachó mi pierna

aa. y se puso a gritar / ¡el niño! ¡el niño! y la huevada ¿cachái?

ab. y yo recién ahí como de despabilé/

ac. me miro y ooh

ad. ahí recién me puse así como ya

ae. y me cagué de miedo po huevón

af. y llega mi mamá

ag. me ve

ah. y partimos a la posta rajados/ huevón/ así brígido $^{10}</$ acción de complicación>

ai. y esa huevada es lo más terrible que me ha pasado así (...) (ESECH: BIHO4).

10 En español de Chile, situación escalofriante. 
En la narración que acabamos de citar, hay una serie de elementos de carácter orientacional, como "un huevón de dieciocho años y yo teniendo ocho años", "y hay un ventanal de vidrio", "pero yo soy tan terco y de chico lo he sido" y "soy orgulloso", que contribuyen a comprender el desarrollo de los sucesos narrados. El resto de las cláusulas es parte de la complicación de la narración. De hecho, algunas de ellas aparecen encabezadas por verbos en presente histórico, $b, e, f, v, w, c c, f f$, gg, lo que permite dinamizar los sucesos y presentarlos como si estuvieran ocurriendo ante los ojos del interlocutor. La partícula de repente, en este caso, se ubica en la primera cláusula narrativa, que presupone el verbo "haber", es decir, de repente está introduciendo la existencia del antagonista de los hechos. A partir de la presentación de la existencia de un segundo involucrado en la historia, se desarrolla una serie de eventos que son relevantes para la secuencia de sucesos. Consiguientemente, se trata de hechos inesperados o dramáticos, desde la perspectiva de SilvaCorvalán (2001).

Junto con lo anterior, es interesante cómo, en muchas ocasiones, el clímax de la historia aparece encabezado por la construcción "y de repente” más un verbo en presente histórico $(\mathrm{PH})$, como veremos en ejemplos posteriores. Por supuesto, hay muchos recursos evaluativos en el marco de las cláusulas correspondientes al punto máximo del relato, y el presente histórico es uno de ellos ( $c f$. Guerrero 2014a y 2014b).

(6) $\quad(. .)<$. resumen $>$ porque cuando vivía en $\mathrm{X}$ entraban a robar $</$ resumen $>$

a. <orientación> cuando yo ya tenía / no sé / ponte tú / estaba en tercero medio

b. tenía como dieciséis

c. entraron a mi

d. o sea/ habían hartos robos </orientación $>$

e. <acción de complicación > y entraron a mi casa 
f. entró un gallo a / no sé / en pelotas ${ }^{11}$ desnudo / a / como a violarme // ¿cachái? // terrible / huevón / pero terrible // E: pero // ¿en la noche?

g. I: sí y me espiaba de antes// E: no// A1: ¿en serio?

h. I: te juro

i. me espiaba el desgraciado

j. y después entró en pelota

k. y ese día fue a la estar a ver tele

1. porque por lo general yo pintaba con lo con mi cómo se llama / con mi walkman

m. dibujaba/ no / un desastre // E: y cuándo / pero / ya / eso era / un tipo degenerado no más

n. I: si pero habían hartos

o. <orientación > igual habían hartos robos

p. habían

q. sí po no fue el único degenerado por ahí

r. porque una vez se le perdió uno de / los perros a mi mamá / el perro más chico/ eh perro / un poodle enano

s. y ya po / Z // se llamaba

t. y Coñac lo fueron a buscar por allá por

u. eh / o sea llegaron hasta X bajando

v. y caminando

w. y X es súper oscuro

$\mathrm{x}$. entonces eh llamaban ellas

y. mi mamá y mi hermana / llamaban $<$ /orientación $>$

z. <acción de complicación > y / y de repente pasa un cabro así como súper bien / para un lado

aa. y después se devuelve

ab. y se pone debajo del farol y el gallo ${ }^{12}$

ac. se baja el pantalón

ad. y empieza a masturbarse

11 Desnudo

12 Hombre joven 
ae. y mi ma/ y mi hermana chica / y mi hermana que ya tenía no sé po / ponte tú / ya tenía quince / y / o / trece / le dice mamá / mira ese gallo//

af. mi mamá lo ve

ag. y le dice mira concha de tu madre ${ }^{13}$

ah. tengo aquí un cuchillo

ai.y te voy a cortar el pico </acción de complicación $>$

aj. <resolución $>$ y el huevón hizo así / shup rajó </resolución $>$

ak. y después la /mi hermana C le decía ¿qué cuchillo mamá?

al.cállate/ mierda

am. eran puras mentiras

an. es que mi mamá es chora

ao. es súper chora

ap. si cuando / cuando / si cuando / el gallo entró empe/ en pelota

aq. y / y lo vió una tía

ar. porque ni siquiera lo vi yo // y mi

as. y el gallo se masturbaba

at. mientras miraba

au. mi tía no podía entender (...) (ESECH: MAIM158).

En el ejemplo que precede puede observarse que entre $p$ y $p p$ hay una historia subordinada al relato principal, que hace referencia a la presencia de un hombre desnudo en el dormitorio de una de las hijas de los dueños de casa. Por su parte, la historia incrustada hace alusión al hecho de que una vez que salieron a buscar a una de sus mascotas, también son sorprendidas por un hombre desnudo. Esta historia, como puede observarse, está perfectamente bien constituida en términos de estructura narrativa, y su presencia no interfiere en el desarrollo de la historia más global, que desde $q q$ continúa narrándose sin inconvenientes. Observamos que de repente introduce la complicación de la narración de la historia incrustada y es tal su valor que "súbitamente" se refuerza con un verbo en presente histórico "de repente pasa” en lugar de "de repente pasó".

13 En Chile, insulto 
Silva-Corvalán (2001) pone de relieve el valor retórico del presente histórico, que coocurre con la expresión de repente. En particular, el presente histórico, al alternar con formas verbales en pretérito, conlleva mayores efectos de viveza y dramatismo, cumpliendo una función evaluativa dentro del relato (Silva-Corvalán 1983).

\subsection{Análisis de de repente en correlación con los factores sociales en el habla de Santiago de Chile}

En este apartado revisaremos, en detalle, el empleo de la partícula de repente en correlación con los factores sociales sexo, edad, grupo socioeconómico y nivel educaciona ${ }^{14}$ de los informantes. Con respecto a esto, los datos que se muestran a continuación corresponden al análisis de las 300 narraciones que conforman la muestra. El único factor externo que se expone de manera independiente según el corpus es el nivel educacional versus el grupo socioeconómico.

Gráfico 1. Porcentaje de frecuencias de empleo de la partícula de repente como introductor de complicación de la narración en relatos de experiencia personal

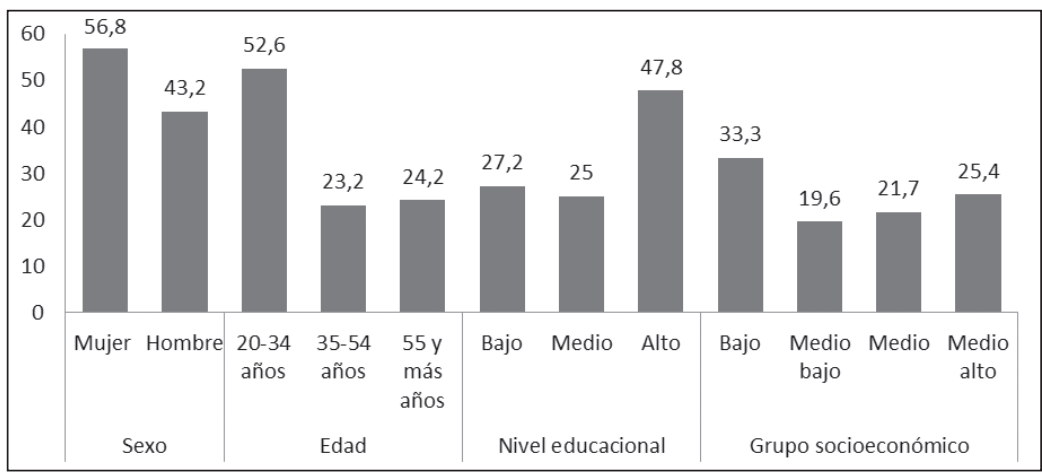

14 Recordemos que ESECH trabaja con la variable externa grupo socioeconómico, determinado por el cruce de tres variables: nivel educacional, profesión u ocupación y comuna de residencia, dando origen a cuatro grupos: bajo, medio bajo, medio y alto, mientras que PRESEEA solo trabaja con la variable nivel educacional, originando tres niveles: bajo, medio y alto, según consta en la Metodología de esta investigación. 
El Gráfico 1 da cuenta de que, en relación con los factores sociales, el empleo de de repente como introductor de complicación de la narración, es utilizado, según el factor sexo, mayoritariamente por las mujeres de la muestra, pues ellas lo utilizan en el 56,8\% (54/65) de los casos, mientras que los hombres lo emplean en el $43,2 \%$ (41/95) de las oportunidades en que aparece con la función descrita. Este hallazgo es de suma relevancia si recordamos que de repente funciona para introducir hechos dramáticos y podría explicarse con la distinción que Hall (1995) postula entre el dominio público y el privado, que instala a los hombres en la esfera de los negocios, del comercio y de la política, y a las mujeres en el mundo privado de la familia y del hogar. Así, en el caso del habla femenina, más que el intercambio de información, lo esencial es el establecimiento y mantenimiento de las relaciones sociales. En consecuencia, parece oportuno vincular el hecho de que las mujeres incluyan en sus historias recursos "dramáticos", dotándolas de mayor dinamismo y vivacidad. Coincidimos, por ende, con los postulados de Prieto y San Martín (2002-2003).

En el caso del factor edad, de repente como introductor de la complicación de la narración se ubica mayoritariamente en las narraciones de los informantes jóvenes de 20 a 34 años, a saber, en el 52,6\% (50/95) de las ocasiones. Por su parte, el segundo y el tercer grupo de edad lo emplean de manera casi idéntica, con $23,2 \%$ (22/95) y $24,2 \%$ (23/95), respectivamente, del total de casos observado en la muestra de estudio.

El nivel educacional, medido solo en el corpus PRESEEA de Santiago de Chile, señala que son los hablantes del grupo alto, es decir, los que tienen educación universitaria en curso o completa, quienes usan con mayor frecuencia esta partícula para introducir el clímax de la historia, con el 47, 8\% (21/44). ${ }^{15}$ En tanto, los grupos bajo y medio lo emplean con una frecuencia similar, a saber, $27,2 \%$ $(12 / 44)$ y $25 \%(11 / 44)$, de manera respectiva. El fenómeno intere-

15 Como señalamos en 4.1. del total de casos, 44/95 (46, 3\%) corresponden al corpus PRESEEA de Santiago de Chile y 51/95 (53, 7\%) al corpus ESECH. 
sante se produce al contrastar esta variable externa con la variable grupo socioeconómico de ESECH, que considera como uno de sus factores clave el nivel educacional, debido a que, como se puede apreciar en el gráfico precedente, es el grupo bajo el que concentra la mayor cantidad de casos de de repente como introductor de complicación de la narración, a saber, un 33,3\% (17/51) del total de usos. Le sigue, en orden de frecuencia, el grupo medio alto con un $25,4 \%$ (13/51), el grupo medio con un $21,7 \%(11 / 51)$ y el grupo medio bajo con 19,6\% (10/51) del total de casos observado en la muestra. Pese a que estos hallazgos parecen, en cierta medida, contradictorios con los expuestos en relación con el vínculo entre el uso de de repente en el corpus PRESEEA de Santiago de Chile con el factor nivel educacional, debemos precisar que los hablantes de los grupos medio alto y alto del corpus ESECH tienen estudios técnicos profesionales completos o universitarios en curso o completos ( $c f$. San Martín y Guerrero 2015), por lo tanto, corresponderían a lo que PRESEEA denomina nivel alto de educación y, en definitiva, la suma de los porcentajes de estos grupos en el empleo de de repente es de 47,1\%, por lo tanto, los datos de ESECH son equivalentes a los proporcionados, en este análisis, por el corpus PRESEEA.

En otro orden, si miramos comparativamente el corpus en estudio en relación con los factores sexo y edad, los datos muestran que se repite en patrón general, al ver cada corpus, en particular. El Gráfico 2 muestra los hallazgos referidos al factor sexo.

Como se desprende del Gráfico 2, en relación con el factor sexo, los datos muestran que, al analizar las muestras por separado, se mantiene la tendencia ilustrada en el Gráfico 1, esto es, que es esta partícula con función de súbitamente suele ser más empleada por mujeres. En concreto, en PRESEEA aparece en 26/44 (59\%) casos y en ESECH se observa en 28/51 (54,9\%) ocasiones. En el caso de los hombres de la muestra, aparece en 18/44 (41\%) en PRESEEA y en $23 / 51(45,1 \%)$ ocasiones en ESECH. Si bien se trata de muestra conformadas por un número diferente de hablantes, lo relevante es que el dato general ( $c f$. Gráfico 1) se repite. 
Gráfico 2. Contraste de porcentaje de frecuencias de empleo de la partícula de repente como introductor de complicación de la narración en relatos de experiencia personal, según el factor sexo en los corpus PRESEEA de Santiago de Chile y ESECH

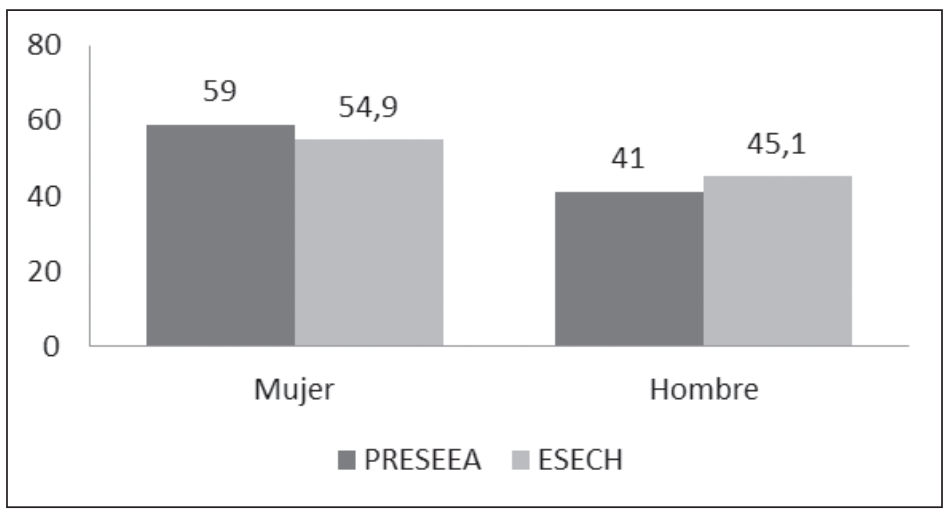

En lo que respecta al factor edad, los hallazgos diferenciados por corpus son los que siguen.

Gráfico 3. Contraste de porcentaje de frecuencias de empleo de la partícula de repente como introductor de complicación de la narración en relatos de experiencia personal, según el factor edad en los corpus PRESEEA de Santiago de Chile y ESECH

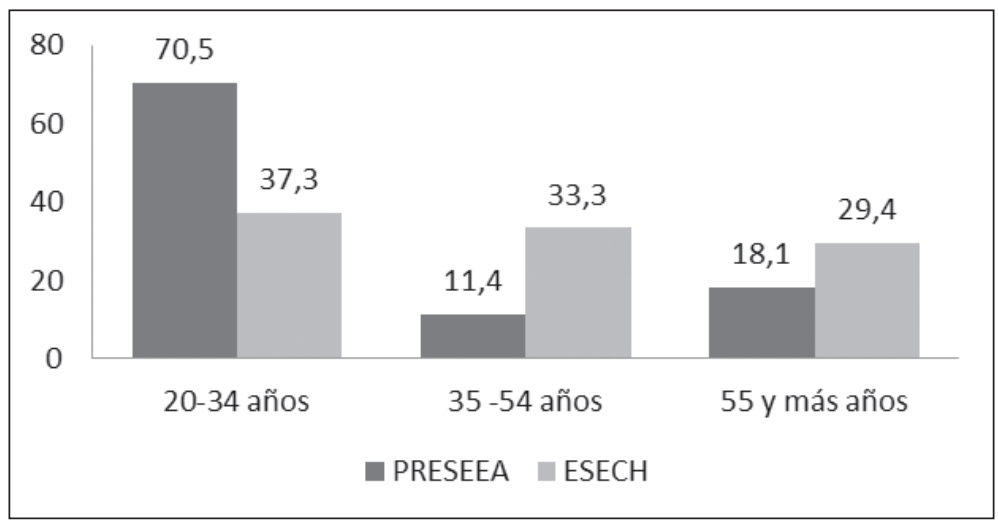


Luego, en el Gráfico 3, se observan los datos contrastivos de los corpus en estudio que, igualmente y pese a la diferencia de informantes que los constituyen, vuelven a reflejar el dato explicitado en el Gráfico 1. Entonces, es el grupo de 20-34 años el que más utiliza esta partícula para introducir la complicación de la narración; en el caso de PRESEEA se usa en 31/44 (70,5\%) oportunidades y en ESECH se emplea en 19/51 (37,3\%) ocasiones. Por su parte, los grupos de 35-54 y de 55 y más años se comportan de manera muy similar en ambos corpus, a saber, en el segundo grupo de edad en PRESEEA se identificaron 5/44 (11,4\%) casos y en ESECH 17/51 $(33,3 \%)$ casos. El tercer grupo etario del corpus PRESEEA, en tanto, usa esta partícula en $8 / 44(18,1 \%)$ oportunidades, mientras que el mismo grupo de edad presenta 15/51 (29,4\%) casos en el corpus ESECH. Las diferencias en ESECH, si bien muestran mayor empleo de esta partícula por el mismo grupo de informantes, no presentan diferencias tan notorias, como en el corpus PRESEEA. Insistimos en que por tratarse de muestras con un número diferente de sujetos por cuota ( $c f$. Tablas 1 y 2), las pruebas debieron realizarse por separado. No obstante, las tendencias apuntan claramente a los mismos hallazgos. La explicación más específica podría hallarse en que, en el corpus ESECH, al haber más hablantes por cuota, podría producirse una disminución en las diferencias de empleo, sumado a la alta tendencia de las mujeres jóvenes de PRESEEA de Santiago de Chile al empleo de de repente para introducir la complicación de la narración.

Junto con los datos proporcionados anteriormente, es relevante destacar que llevamos a cabo pruebas de estadística inferencial, a fin de respaldar los datos porcentuales reportados. Al respecto, nuevamente hay que tener en consideración que, por tratarse de dos corpus con un número distinto de sujetos por cuota, las pruebas se aplicaron de manera independiente a cada corpus. No obstante, el análisis de la totalidad de los datos, expuesto en 4.1. y en el Gráfico 1 de 4.2., nos ayuda a proporcionar una visión más amplia del fenómeno en estudio que, por lo demás, coincide, en su empleo, en el análisis de los corpus de manera independiente. 
En relación con las pruebas estadísticas, es necesario dar cuenta de que los histogramas generados dan cuenta de la dispersión de los datos de la muestra, donde la media aritmética, en PRESEEA de Santiago de Chile es de 0,41 y la desviación típica de 1, 394, y en ESECH la dispersión es de 0,27 y la desviación típica de 0,629, por lo tanto, fue preciso llevar a cabo un análisis de estadística no paramétrica. En este caso, aplicamos una prueba Kruskal-Wallis, que arrojó como resultado que solo el factor edad es estadísticamente significativo para el empleo de de repente como introductor de la complicación de la narración en la muestra PRESEEA $(\mathrm{p}=0,019)$, es decir, esta partícula es empleada por el grupo de edad joven (20 a 34 años) de la población santiaguina. Sin embargo, no se encontraron casos estadísticamente significativos en la muestra ESECH. Este hallazgo podría explicarse debido a las evidentes tendencias en el empleo de de repente por parte de los hablantes del grupo de mujeres de edad joven en el corpus PRESEEA de Santiago de Chile. De manera consecuente, reiteramos la descripción de la mujer como más "teatral” en sus narraciones.

Como señalamos en 4.2. de esta investigación, una de las combinaciones más relevantes para el desarrollo de las narraciones de experiencia personal es la de la partícula de repente + un verbo en presente histórico (PH). En Guerrero (2014b) dimos cuenta de la relevancia que tienen los verbos en presente histórico para la evolución de este tipo de narrativas. Entre otros aspectos, se destacaba el hecho de que el presente histórico pudiera funcionar como un fenómeno variable dentro del discurso narrativo, con una función específica, a saber, la de ser un recurso de evaluación interna dentro del relato. Asimismo, en el mencionado trabajo se concluyó que las cláusulas narrativas suelen estar encabezadas por formas verbales en presente histórico, obedeciendo a lo que Silva-Corvalán (1983) denomina evaluación interna. Consecuentemente, el presente histórico estaría directamente vinculado con los eventos más reportables del relato, otorgándole vivacidad a la historia y justificando la narración de la misma. Así, en la presente investigación nos ha llamado especialmente la atención la manera en que este tipo 
de verbos aparece encabezado por la partícula de repente. En lo que sigue, citamos algunos ejemplos para ilustrar este hallazgo:

(7) (...) y de repente así por acto de magia se metió solo para la casa pues // este huevón retrocedió el auto ¿cachái? / nos quedamos parados en la esquina / de repente llega / el dueño de la casa / llegaron los ratis ${ }^{16}$ estaban los ratis dentro de la casa / en todas las casas que habían / porque era un pasaje sin salida (...) (PRESEEA: M11_011).

(8) (...) y de repente siento que alguien me mueve / ¿cachái? / y despierto como que vuelvo a mi cuerpo ¿cachái? / y despierto ¿cachái? (PRESEEA: H12_041).

(9) (...) entonces cuando de repente miro yo y adelante había un jovencito de pantalones cortos casi / como quien dice un un teenager y yo dije ese niño va adelante seguramente eeh va a tratar de hablar conmigo o o podría ser un asaltante / entonces mejor me voy a abuenar con él / dije yo y me acerqué / adelanté / y me acerqué y conversé con él / nos vinimos conversando / conversando todo el camino hasta acá (...) (PRESEEA: M32_071).

(10) (...) me metieron en una villa que era/ del/ del barrio alto podríamos decir y/ en un pasaje/ donde seguramente todos trabajan porque/ yo sabía no había nadie/ y de repente me veo con cuatro/ con cuatro jah no!/ el personaje que al lado/ me pegó con un tonto de goma en la cara po (...) (ESECH: MB II H067).

(11) (...) y yo fui sola y de repente me meten en un salón enorme// como un auditorio y había un estrado// y me paro y me dice/ ya me dice $\mathrm{X}$ adelante// y iyo creí que me iba a desmayar! (...) (ESECH: M III M140).

En el ejemplo (7) se observa que la partícula en estudio aparece en el clímax de la narración. En el primer caso, funciona como intro-

16 En Chile, Policía de Investigaciones 
ductor de la cláusula narrativa clave de la historia, en tanto que en la segunda ocasión se acompaña de un verbo en presente histórico, "llega" por "llegó", de manera que el suceso se sitúa ante los ojos del interlocutor, debido al traslado del tiempo, es decir, el narrador lo presenta como si estuviese ocurriendo en el momento de la narración. En (8), la partícula de repente también va acompañada de un verbo en presente histórico "siento" y este tiempo verbal se mantiene cuando el hablante pone de relieve "despierto" en lugar de “desperté", en dos ocasiones. Además, en (8) es interesante que de repente aparezca introducido por la conjunción “y”, combinación que suele reiterarse en el corpus en estudio.

En el ejemplo (9) de repente va acompañado del verbo "mirar", que adopta la forma de presente histórico "de repente miro". En este caso, además, en lugar de la conjunción copulativa “y”, la partícula en estudio va introducida por la conjunción temporal "cuando", que es también frecuente en la combinación de repente + PH. Por su parte, en (10) se emplea la forma "de repente me veo" en lugar de "de repente me vi”, que es la que esperaríamos si se están contando hechos ocurridos en el pasado. Asimismo, vuelve a aparecer la conjunción "y" para marcar la presencia de "de repente". Por último, en (11) se repite la combinación "y de repente $+\mathrm{PH}$ ”. En este ejemplo, además, los verbos en presente histórico se conservan a medida que se desarrolla la complicación de la narración “me paro" y "me dice”, en lugar de "me paré” y “me dijo”.

Es relevante destacar que futuras investigaciones podrían centrarse en el estudio de las partículas discursivas que introducen a la forma de repente. En este trabajo solo las describimos someramente, porque no forma parte de nuestros objetivos básicos.

Con el propósito de complementar este dato, hemos realizado el análisis porcentual correspondiente, lo que nos ha permitido comprobar que de los 95 casos en los que se emplea de repente para introducir complicación de la narración, 42/95 (44,2\%) responden a la colocación de repente $+\mathrm{PH}$, es decir, en cerca de la mitad de la muestra se utiliza esta estrategia discursiva. Por su parte, la relación 
de estos datos con las variables externas en estudio es la que se detalla en el Gráfico 4, que presentamos a continuación.

Gráfico 4. Porcentaje de frecuencias de empleo de la partícula de repente $+\mathrm{PH}$ como introductor de complicación de la narración en relatos de experiencia personal

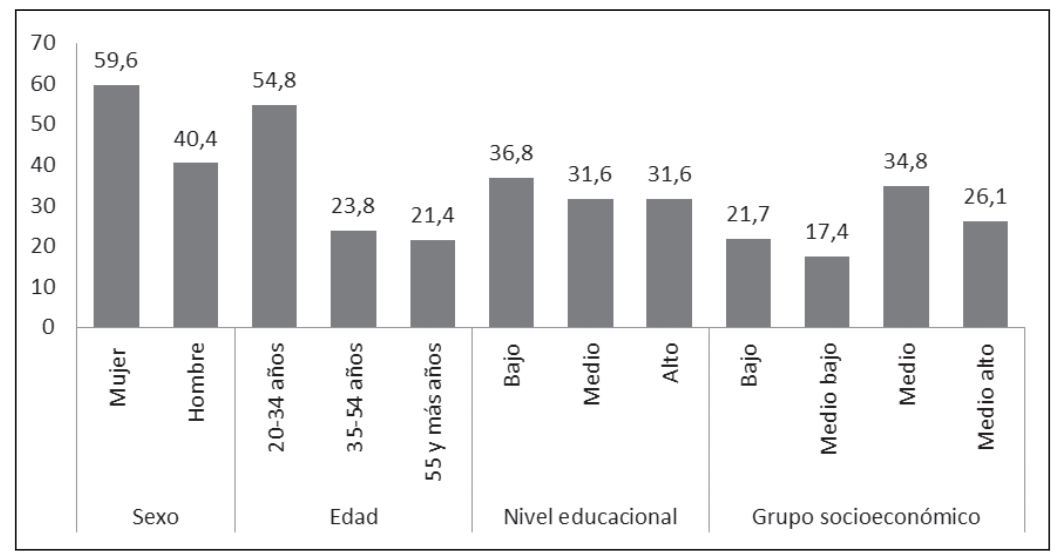

Como se desprende del Gráfico 4, los datos coinciden con los expuestos en el Gráfico 1, en los factores sexo y edad. Además, son semejantes a los expuestos, en términos porcentuales, en Guerrero (2014b) respecto de estas mismas variables externas. De manera consecuente y tomando en consideración que este dato del trabajo es solo tangencial, solo haremos alusión al análisis de las mencionadas variables externas, que son las que han mostrado datos más relevantes para el desarrollo de este estudio. Así, la colocación ya señalada se da mayormente en narraciones de mujeres, donde se identificaron 25/42 (59,6\%) casos, mientras que en las de los hombres se presentó en 17/42 (40,4\%) oportunidades. En cuanto a la edad, nuevamente es el grupo de 20 a 34 años el que más usa la partícula en estudio con presente histórico; esto ocurre en 23/42 $(54,8 \%)$ ocasiones, en tanto que los grupos de edad intermedia y mayor emplean dicha colocación en $10 / 42(23,8 \%)$ y 9/42 (21,4\%) oportunidades, respectivamente. Asimismo, realizamos las pruebas 
estadísticas no paramétricas correspondientes y comprobamos que en el corpus PRESEEA de Santiago de Chile, dicha colocación es estadísticamente significativa en su empleo en correlación con el factor edad, donde $\mathrm{p}=0,007$, tal como ocurrió con el análisis del empleo general de la partícula en estudio.

Esta colocación había sido descrita por Parra (2012), quien analizó narraciones orales de carácter autobiográfico. La autora señala, al respecto, que:

Una marca que indica claramente la presencia de un acontecimiento que sorprende y afecta al hablante, es el uso de la locución adverbial de repente para introducir el $\mathrm{PH}$, ya que este se clasifica como un adverbio de modo utilizado para referirse a eventos que ocurren súbitamente, sin preparación (Parra 2012: 70).

Como se desprende de los ejemplos que hemos citado con anterioridad, en nuestro corpus son numerosos los casos en los que de repente corresponde a lo que Rojas (2008) denomina DR1, es decir, a una partícula que enuncia segmentos de alto dinamismo narrativo. En otras palabras, lo que Parra (2012) destaca como un acontecimiento que resulta sorpresivo para el hablante y que, a su vez, corresponde al tipo de relato que Labov (1972) considera digno de contarse.

Para finalizar esta investigación, hemos querido hacer una breve revisión del empleo de la partícula de repente en los materiales disponibles del PRESEEA. A este respecto, hemos encontrado, en términos generales, que los tres valores de de repente descritos por Rojas (2008) se presentan en diferentes variedades del español, como muestran los ejemplos (12) al (15).

(12) (...) bueno / muy bueno / porque es como que te cambia todo de repente todo te cambió no para malo ¿no? (La Habana H12_037).

(13) (...) como uno dice en cualquier momento que / bueno / que $d e$ repente es verdad que vuelves y le compras la vaina y de repente no (Caracas H23_085). 
(14) (...) si he estado veinticuatro años viviendo eso y de repente cojo y cambio / y me voy a un sitio tranquilo (Valencia H13_020).

(15) (...) vos simplemente digo no / si te interesa / de repente no querés integrar un grupo eh / y te interesa el (...) (Montevideo M22_022).

En (12) la partícula en estudio se utiliza como DR1, en (13) y en (14) como DR2 y en (15) como DR3. Consecuentemente, otra de las proyecciones de esta investigación es la de llevar a cabo estudios contrastivos de las variedades del español respecto de la utilización de este tipo de partícula en la producción oral. Los ejemplos recién citados no fueron tomados necesariamente de secuencias narrativas, lo que podría incidir en que aparezca la función de DR3, que no fue relevada en el corpus de relatos analizado en nuestra investigación. También es pertinente proponer estudios que diferencien las funciones de de repente según los tipos de secuencia discursiva.

\section{Conclusiones}

Las conclusiones más relevantes de nuestra indagación pueden sintetizarse del siguiente modo:

En primer lugar, es destacable que en las 300 narraciones de experiencia personal analizadas, de repente tuvo una frecuencia de 100 casos, de los cuales 95 (95\%) cumplen la función de introducir un hecho inesperado o dramático, tal como lo describe Silva-Corvalán (2001). Se trata, por lo tanto, de la forma que Rojas (2008) denomina DR1 y que equivale a "súbitamente". De esta manera, comprobamos que de repente funciona como introductor de la acción complicada de los relatos. El 5\% restante, en nuestro análisis, fue equivalente a la locución adverbial de frecuencia “a veces”, que se ubicaba en cláusulas orientacionales, en el sentido laboviano.

En relación con el análisis de correlación con los factores sociales, comprobamos que se trata de una partícula que suele emplearse más por informantes mujeres de edad joven (20-34 años). 
Asimismo, de repente como introductor de la complicación de la narración se usa más en informantes con nivel educacional alto o de los grupos socioeconómicos más altos de la escala social. El análisis estadístico, por su parte, da cuenta de que solo el factor edad es estadísticamente significativo, lo que, intuimos, se da por la evidente diferencia en el empleo de esta partícula en el corpus PRESEEA de Santiago de Chile entre los distintos grupos etarios. En concreto, que se trate de una forma discursiva — con función de DR1, según Rojas (2008) - más propia del segmento joven de la población es la conclusión más relevante de este análisis. En consecuencia, hemos comprobado parcialmente nuestra hipótesis de estudio.

En tercer término, el análisis más detallado del empleo de de repente en narraciones de experiencia personal da cuenta de que una colocación preponderante es la que se genera entre la forma de repente + presente histórico, que apareció en 42/95 (44,2\%) del total de casos de la muestra. Este hallazgo es particularmente interesante, porque el presente histórico ha sido descrito como un mecanismo de evaluación interna, que se vincula con los eventos más reportables de la narración (Silva-Corvalán 1983). En Guerrero (2014b) se comprobó que esta forma de pretérito se ubicaba solo en el clímax del relato de experiencia personal y, por lo mismo, podría explicarse su alta frecuencia en combinación con de repente. En este caso también el factor edad mostró se estadísticamente significativo, por lo que podríamos señalar que esta colocación es más frecuente en narraciones de hablantes jóvenes (20-34 años) de la comunidad de habla en estudio.

Por último, se proponen como proyecciones del trabajo el análisis de las partículas discursivas que introducen a la forma de repente, pues hemos encontrado que al parecer se da, en muchos casos, con "y" y "cuando". Asimismo, sugerimos realizar estudios contrastivos del empleo de esta partícula con otras variedades del español y, finalmente, proponemos ver su funcionamiento en distintos tipos de secuencias discursivas. 


\section{Corpus consultados}

\section{PRESEEA}

2014 Corpus del Proyecto para el estudio sociolingüístico del español de España y de América. Alcalá de Henares: Universidad de Alcalá. Consultado: 19 de septiembre de 2015. <http://preseea. linguas.net>.

2014 Corpus del grupo de Estudio Sociolingüistico del Español de Chile (ESECH). Coords., Abelardo San Martín Núñez y Silvana Guerrero González. Santiago: Universidad de Chile.

\section{Referencias bibliográficas}

Academia Chilena de la Lengua

2010 Diccionario de uso del español de Chile (DUECh). Santiago: MN Editorial.

Alarcos Llorach, Emilio

1999 Gramática de la lengua española. Madrid: Espasa Calpe.

Bassols, Margarida y Ana Torrent

2003 Modelos textuales. Barcelona: Eumo-Octaedro.

Becerra, Rukmini, Daniela Molina, Darío Rojas y María Antonieta Vergara 2005 Estratificación social del empleo de los marcadores discursivos en el habla de Santiago de Chile. Tesis de Licenciatura. Santiago: Universidad de Chile. Consultado: s/f. <http://www. repositorio.uchile.cl/handle/2250/110194>.

Blas Arroyo, José Luis

2005 Sociolingüística del español. Desarrollos y perspectivas en el estudio de la lengua española en contexto social. Madrid: Cátedra.

Briz, Antonio, Salvador Pons y José PORTOlÉs (coords.)

2008 Diccionario de partículas discursivas del español. Consultado: s/f. <http://www.dpde.es>.

Coates, Jennifer

2009 Mujeres, hombres y lenguaje. Un acercamiento sociolingüístico a las diferencias de género. México: Fondo de Cultura Económica. 
Gili GayA, Samuel

1961 Curso superior de sintaxis española. Barcelona: Spes.

GonZÁlez, Consuelo

2013 "Uso de por ejemplo, por lo menos, de repente y otros operadores de concreción en el español hablado de Caracas”. Boletín de Lingüistica. XXV, 39-40, 61-91.

Guerrero GonzÁlez, Silvana

2014a Variación discursiva en narraciones de experiencia personal en el español hablado en Santiago de Chile. Tesis doctoral inédita. Santiago: Pontificia Universidad Católica de Chile.

2014b "Una aproximación sociolingüística al empleo del presente histórico en narraciones de experiencia personal en hablantes de Santiago de Chile". RLA Revista de lingüística teórica y aplicada. 52, 2, 89-112. https://doi.org/10.4067/ s0718-48832014000200005

Hall, Kira

1995 "Lip service on the fantasy lines". En Gender articulated: Language and the constructed self. Eds., K. Hall y M. Bucholtz. Nueva York y Londres: Routledge, 183-216.

Hernández Campoy, Juan Manuel y Manuel Almeida

2005 Metodología de la investigación sociolingüística. Málaga: Editorial Comares.

JIMÉNEZ, Teira

2006 "La narración infantil. Un estudio en niños de educación básica”. Revista de investigación. Universidad Central de Venezuela. 60, 157-174.

Johnstone, Barbara

2006 "A new role for narrative in variationist sociolinguistics". Narrative Inquiry.1,46-55. https://doi.org/10.1075/ni.16.1.08joh

LABOV, William

1972 "The transformation of experience in narrative syntax". En Language in the inner city. Studies in the black English vernacular". Filadelfia, Estados Unidos: University of Pennsylvania Press, 354-375.

1983 [1972] Modelos sociolingüísticos. Madrid: Cátedra. 
LABOV, William y Joshua WALETZKY

1967 "Narrative analysis". En Essays on the verbal and visual arts. Ed., J. Helm. Seattle: University of Washington Press, 12-44.

LENSKI, Gerhardt

1954 "Status Crystallization: A Non-vertical Dimension of Social Status". American Sociological Review. 19, 405-413. https:// doi.org/10.2307/2087459

López Morales, Humberto

1994 Métodos de investigación lingüística. Salamanca: Ediciones Colegio de España.

Moreno Fernández, Francisco

1990 Metodología sociolingüística. Madrid: Gredos.

1998 Principios de sociolingüística y sociología del lenguaje. Barcelona: Ariel.

2009 Principios de sociolingüística y sociología del lenguaje. $4^{\mathrm{a}}$ ed. Barcelona: Ariel.

2012 Sociolingüística cognitiva. Proposiciones, escolios y debates. Madrid/Frankfurt: Iberoamericana/Vervuert.

PARra, Gabriela

2012 Análisis del presente bistórico y sus funciones en narraciones orales de carácter autobiográfico. Tesis para optar al grado de Magíster en Estudios cognitivos. Santiago: Universidad de Chile.

PORTOLÉs, José

2001 Marcadores del discurso. $2^{\mathrm{a}}$ ed. Barcelona: Ariel.

PRIETO, Luis

1995-1996 "Análisis sociolingüístico del dequeísmo en el habla de Santiago de Chile”. Boletín de Filología. XXXV, 379-452.

Prieto, Luis y Abelardo SAN MARTín

2002-2003 "Diferencias de género en el empleo del discurso referido: aproximación sociolingüística y pragmático-discursiva”. Boletín de Filología. XXXIX, 269-304.

Real Academia Española

2001 Diccionario de la lengua española. 22ª ed. Madrid: España. 
2010 Nueva gramática de la lengua española. Manual. Madrid: Espasa Libros.

Reyes, Claudia

1996 "Estrategias narrativas en la zona metropolitana de Monterrey". En Lenguaje y sociedad: Metodología y análisis aplicados a El habla de Monterrey. Eds., Dora Esthela Rodríguez Flores y Lidia Rodríguez Alfano. México: Trillas, 101-120.

2002 "Algunas diferencias en las narraciones de hombres y mujeres dentro del corpus de 'El habla de Monterrey'”. Iztapalapa. 53, 101-115.

2003 "Visión panorámica de los estudios sobre la narración". Revista de Humanidades: Tecnológico de Monterrey. 15. Instituto Tecnológico y de Estudios Superiores de Monterrey (ITESM): México, 95-119.

Rojas, Darío

2008 "Funciones actuales y evolución semántica de la locución de repente en el español de Chile”. Boletín de Filología. 43, 1, 207237.

San Martín, Abelardo y Silvana Guerrero

2015 "Estudio sociolingüístico del español de Chile (ESECH): recogida y estratificación del corpus de Santiago". Boletín de Filología. L, 1, 221-247. https://doi.org/10.4067/s071893032015000100009

SERrano, María José

2011 Sociolingüística. Barcelona: Ediciones del Serbal.

SHIRO, Martha

2003 "Genre and Evaluation in Narrative Development. Journal of Child". Language. 30, 1, 165-195. https://doi.org/10.1017/ s0305000902005500

Silva-Corvalán, Carmen

1983. "Tense and aspect in oral Spanish narrative: Context and meaning”. Language. 59, 4, 760-780. https://doi.org/10.2307/ 413372

2001 Sociolingüistica y pragmática del español. Washington: Georgetown University Press. 
SOLER, Sandra

2004 Discurso y género en historias de vida. Una investigación de relatos de hombres y mujeres en Bogotá. Bogotá: Publicaciones del Instituto Caro y Cuervo.

Fecha de recepción: 20/10/2015

Fecha de aceptación: 30/05/2016 